\title{
Auszug aus der S1-Leitlinie Intermittierende pneumatische Kompression (IPK, AIK)
}

\section{S. Reich-Schupke; C. Schwahn-Schreiber}

Im Namen der Autorengruppe der Leitlinie: Dr. Franz-Xaver Breu, Prof. Dr. Eberhard Rabe, Prof. Dr. Ivo Buschmann, Dr. Walter Döller, Prof. Dr. Gerd Lulay, Dr. Anya Miller, PD Dr. Eva Valesky

\section{Schlüsselwörter \\ Kompression, Ödem, Entstauung, Perfusion}

\section{Zusammenfassung}

Die Intermittierende pneumatische Kompressionstherapie (IPK, AIK) besteht in der prophylaktischen und therapeutischen Anwendung von pneumatischen Wechseldrücken zur Thromboembolieprophylaxe, Entstauungstherapie bei Ödemerkrankungen, Verbesserung der arteriellen und venösen Durchblutung mit Reduktion der klinischen Symptome sowie zur Förderung der Wundheilung. Die Therapie kann sowohl stationär als auch ambulant in einer medizinischen Einrichtung oder - nach entsprechender Schulung - als Heimtherapie angewendet werden. Die Geräte bestehen aus Generator

Korrespondenzadresse

Prof. Dr. Stefanie Reich-Schupke

Klinik für Dermatologie, Venerologie und Allergologie

Venenzentrum der Dermatologischen und Gefäßchirurgischen Kliniken

Stiftungsprofessur Phlebologie

Gudrunstr. 56

44791 Bochum

Tel. 0234-8792-377 / -274

E-Mail: Stefanie.Reich-Schupke@rub.de und Manschetten und unterscheiden sich abhängig von ihrer Indikation und der Ziellokalisation im Aufbau sowie in den technischen Parametern der Therapie. Bei richtiger Indikationsstellung und Anwendung handelt es sich um eine effektive und sichere Therapiemethode. Unerwünschte Ereignisse treten extrem selten auf. Es besteht ein dringender Bedarf an methodisch gut gemachten Studien zur IPK in verschiedenen Indikationen (Ausnahme VTE-Prophylaxe, gute Evidenz). In den Protokollen sollten zwingend genaue Angaben zum verwendeten Gerät (Kompression und Manschette) sowie zum Behandlungsprotokoll (Inflation, Deflation, Plateau, Pausenzeiten, Behandlungsfrequenz pro Tag oder Woche sowie zur Gesamtdauer der Anwendung) gemacht werden.

\section{Zitierweise des Beitrags:}

Abstract of the S1-Guideline: Intermittend pneumatic compression (IPK, AIK)

Phlebologie 2018; 47: 272-275

https://doi.org/10.12687/phleb2422-5-2018

Eingegangen: 04. Juli 2018

Angenommen: 08. Juli 2018

English version available at: www.phlebologieonline.de
Keywords

Compression, congestion, edema, perfusion

Summary

The intermittend pneumatic compression (IPK, AIK) consists of prophylactic or therapeutic use of alternating pressure for prophylaxis of thrombosis, treatment of edema, improvement of arterial and venous perfusion with reduction of clinical symptoms and improvement of wound healing. IPK can be used in an in- and outpatient setting as well as a home treatment after education of the patient. The equipment consists of a generator and additional cuffs. The devices differ according to indication, localisation of treatment and technical parameters. With correct use and indication, IPK is an effective and safe treatment option. Adverse events are very rare. There is an urgent need for methodical well conducted studies in different indications - except prophylaxis of venous thromboembolia. The protocols should list exact data on the used device (compression and cuffs) and technical parameters (inflation, deflation, plateau, time of break, use per day or week, duration of use).

\section{Wichtiger Hinweis!}

Das aktuelle Manuskript stellt eine Zusammenfassung wesentlicher Empfehlungen der Leitlinie dar. Es handelt sich NICHT um die komplette Version. Diese finden Sie online unter https://www.awmf.org/uplo ads/tx_szleitlinien/037-0071_S1_Intermittierende-pneumatische-Kompression-IPKAIK_2018-05.pdf (Zugriff 03.07.2018) sowie zitierfähig unter Schwahn-Schreiber, C., Breu, F.X., Rabe, E. et al. S1-Leitlinie In- termittierende Pneumatische Kompression (IPK, AIK). Hautarzt (2018). (https://doi. org/10.1007/s00105-018-4219-1

(https://link.springer.com/arti cle/10.1007/s00105-018-4219-1; Zugriff 03.07.2018). In den angegebenen Quellen finden Sie auch die Literaturliste.

\section{Einleitung}

Die Überarbeitung der Leitlinie erfolgte in einer Expertenkommission bestehend aus Mitgliedern verschiedener Fachgesellschaften mit dem Ziel, die Indikationsstellung und therapeutische Anwendung der IPK bei Gefäß- und Ödemerkrankungen zu optimieren. Die Ausführungen und Empfehlungen wurden nach intensiver Literaturrecherche und im Expertenkonsensus erarbeitet. In einigen Themenfeldern bot die 
Tab. 1 Indikationen für die IPK

Bei folgenden Indikationen soll eine IPK erfolgen:

- Thromboembolieprophylaxe, wenn keine medikamentöse Prophylaxe möglich ist

Bei folgenden Indikationen sollte eine IPK erfolgen:

- Ulcus cruris venosum mit fehlender Heilungstendenz trotz konsequenter Kompressionstherapie mittels Strumpfsystemen oder Kompressionsverbände

- Schwere chronische venöse Insuffizienz im Stadium C4b bis C6 (CEAP Klassifikation)

Extremitätenlymphödem, additiv bei fehlender Kompensation unter komplexer physikalischer Entstauungstherapie

- Periphere arterielle Verschlusskrankheit (pAVK) mit stabiler Claudicatio intermittens oder kritischer Ischämie, wenn ein angeleitetes Übungsprogramm nicht möglich ist und eine interventionelle oder operative Rekonstruktion nicht in Frage kommt

Bei folgenden Indikationen kann eine IPK erfolgen:

- Posttraumatische Ödeme

- Therapieresistente venös bedingte Ödeme

- Lipödem

- Extremitätenlymphödem, additiv zur komplexen physikalischen Entstauungstherapie

- Hemiplegie mit sensorischer Störung und Ödem

- Thromboembolieprophylaxe zusätzlich zur medikamentösen Prophylaxe

Literatur nur wenig Material, so dass die Empfehlungen entsprechend der Erfahrungen des klinischen Alltags ausgesprochen wurden. Auch in den Leitlinien zur Thromboembolieprophylaxe, zum Lymphödem und Lipödem gibt es eigene $\mathrm{Ab}$ schnitte zur IPK. Diese sollten bitte zusätzlich beachtet werden.

\section{Definition und Wirkung}

Das Prinzip der IPK besteht in der prophylaktischen und therapeutischen Anwendung von pneumatischen Wechseldrücken zur Thromboembolieprophylaxe, Entstauungstherapie bei Ödemerkrankungen, Verbesserung der arteriellen und venösen Durchblutung mit Reduktion der klinischen Symptome sowie zur Förderung der Wundheilung. Die Therapie kann sowohl
Tab. 2 Kontraindikationen für die IPK

- Bei Vorliegen einer absoluten Kontraindikation soll keine IPK durchgeführt werden.

- Bei Vorliegen einer relativen Kontraindikation kann unter engmaschiger klinischer Kontrolle und entsprechenden Vorsichtsmaßnahmen eine IPK durchgeführt werden (siehe Nebenwirkungen).

Die folgenden absoluten Kontraindikationen sollen beachtet werden:

- Dekompensierte Herzinsuffizienz

- Ausgedehnte Thrombophlebitis, Thrombose oder Thromboseverdacht

- Akutes Erysipel

- Akute Phlegmone

- Kompartmentsyndrom

- Schwere, nicht eingestellte Hypertonie

- Daneben soll bei okkludierenden Prozessen im Lymphabstrombereich, bei denen es unter IPK zu einem Stau im Leistenbereich oder Genitalbereich gekommen ist, keine IPK durchgeführt werden

Die folgenden relativen Kontraindikationen sollen beachtet werden:

- Ausgedehntes, ggf. offenes Weichteiltrauma der Extremitäten

- Ausgeprägte Neuropathie der Extremitäten

- Blasenbildende Dermatosen wie z. B. IgADermatitis oder Pemphigoid

stationär als auch ambulant in einer medizinischen Einrichtung oder - nach entsprechender Schulung - als Heimtherapie angewendet werden. Die eingesetzten Geräte unterscheiden sich abhängig von ihrer Indikation und der Ziellokalisation.

Die Wirkung der IPK ist dabei mannigfaltig. Unter anderem wirkt sie hämodynamisch, hämatologisch, fibrinolytisch, beeinflusst die Sauerstoffspannung im Gewebe und reduziert ein Ödem.

\section{Indikationen und Kontraindikationen}

Entsprechend der aktuellen Studienlage ergeben sich die in Tabelle 1 genannten Empfehlungen für den Einsatz der IPK. Vor einem Einsatz sollte eine exakte Diagnosestellung erfolgen und initial sowie im Verlauf auf mögliche Kontraindikationen $($ Tab. 2) geachtet werden.
Tab. 3 Zur Vermeidung von Nebenwirkungen und Komplikationen wird empfohlen

- Zur Vermeidung von Hautschäden sollte ein textiler Hautschutz unter der Kunststoffmanschette eingesetzt und eine regelmäßige Inspektion und Pflege der Haut durchgeführt werden.

- Zur Verhinderung von Nervenschädigungen und Drucknekrosen sollte insbesondere bei schlanken oder kachektischen Patienten eine Polsterung über Dispositionsstellen erfolgen.

- Genitallymphödeme wurden in keiner Studie seit 1998 beschrieben bzw. explizit verneint. Es sollte darauf geachtet werden. Wenn es unter der IPK beim Lymphödem zu einer Zunahme des Ödems im Becken- oder Genitalbereich kommt, soll auf eine weitere Durchführung der IPK verzichtet und die Diagnostik bezüglich eines Abflusshindernisses erweitert bzw. wiederholt werden.

\section{Risiken und Nebenwirkungen}

Entsprechend der aktuellen Datenlage mit nur wenigen Fallberichten und einer retrospektiven Erhebung zu entsprechenden Nebenwirkungen und Komplikationen kann die IPK als effektiv und sicher eingeschätzt werden ( Tab. 3). Die beschriebenen Hautschädigungen traten v.a. unter mehrtägiger, kontinuierlicher Anwendung der IPK im Rahmen der VTE-Prophylaxe auf. Beschrieben wurden Hautläsionen, Follikulitiden und Blasenbildungen v.a. bei älteren Patienten mit fragiler Haut. Es gab Weichteilschädigungen bei falscher Anlage sowie Nervenschädigungen, Drucknekrosen und sehr selten ein Kompartmentsyndrom oder eine Lungenembolie. In einer älteren retrospektiven Studie wurde die Ausbildung von genitalen Lymphödemen beobachtet. Studien der letzten 15 Jahre haben ein Genitallymphödem bzw. fibrosierte Geweberinge im Leistenbereich nicht beschrieben bzw. explizit verneint. Es sollte aber darauf geachtet werden. Bei empfindlichen Hautverhältnissen wie z.B. ausgeprägter Stauungsdermatose oder lymphokutanen Fisteln sollte die IPK bis zur Hautstabilisierung nur unter antibiotischem Schutz durchgeführt werden. 
Tab. 4 Empfehlungen zum Einsatz der IPK bei den verschiedenen Indikationen im Überblick.

\begin{tabular}{|c|c|}
\hline Indikation & Empfehlungen \\
\hline $\begin{array}{l}\text { Thromboembolieprophylaxe (Die } \\
\text { Empfehlungen zur Thromboseprophy- } \\
\text { laxe beziehen sich auf die S3-Leitlinie } \\
\text { Prophylaxe der venösen Thromboem- } \\
\text { bolie (VTE) und wurden hier zur bes- } \\
\text { seren thematischen Übersicht und zur } \\
\text { Vollständigkeit zusammenfassend } \\
\text { dargestellt. Folgende Kernempfehlun- } \\
\text { gen seien hier aus dieser Leitlinie } \\
\text { übernommen) }\end{array}$ & $\begin{array}{l}\text { Bei Patienten mit mittlerem und hohem VTE-Risiko sollten neben einer medikamentösen Prophylaxe Basis- } \\
\text { maßnahmen eingesetzt werden. } \\
\text { - Zusätzlich können physikalische Maßnahmen angewendet werden. } \\
\text { Basismaßnahmen sowie physikalische Maßnahmen sollen eine indizierte medikamentöse VTE-Prophylaxe } \\
\text { nicht ersetzen. } \\
\text { - Umgekehrt sollte bei einer medikamentösen VTE-Prophylaxe nicht auf Basismaßnahmen verzichtet und phy- } \\
\text { sikalische Maßnahmen sollten indikationsgerecht eingesetzt werden. } \\
\text { Bei Kontraindikationen gegen eine medikamentöse VTE-Prophylaxe sollen physikalische Maßnahmen zur An- } \\
\text { wendung kommen. } \\
\text { Es erscheint sinnvoll, dass jedes Krankenhaus einige IPK-Geräte vorhält, um das Verfahren bei Patienten mit } \\
\text { hohem VTE-Risiko und Kontraindikation gegen eine medikamentöse Prophylaxe zur Anwendung bringen zu } \\
\text { können. }\end{array}$ \\
\hline $\begin{array}{l}\text { Chronische venöse Insuffizienz mit } \\
\text { und ohne Ulcus cruris venosum }\end{array}$ & $\begin{array}{l}\text { Bei Patienten mit CVI kann die IPK eingesetzt werden, um chronisch venöse Symptome und die Lebensquali- } \\
\text { tät zu verbessern. } \\
\text { - Die IPK kann bei Patienten mit CVI als Heimtherapie eingesetzt werden. } \\
\text { Die IPK bei CVI ohne Ulcus cruris sollte mit Druckwerten von 30-40 mmHg, einer Inflation von 15s und einer } \\
\text { Deflation von 10s mit mehrstufigen Manschetten und einem Druckaufbau von distal nach proximal erfolgen. } \\
\text { - Die IPK (Bein) sollte bei Patienten, deren Ulcus cruris venosum unter Standardtherapie keine Heilungsten- } \\
\text { denz zeigt, mit mehrstufigen Manschetten (sequentieller Druckaufbau, Zieldruck } 40 \text { - } 50 \text { mmHg, mindestens } \\
1 \text { Stunde pro Tag, mindestens } 3 \text { x pro Woche) zur Wundheilungsförderung eingesetzt werden. }\end{array}$ \\
\hline Posttraumatische Ödeme & $\begin{array}{l}\text { Bei Patienten mit posttraumatischem Ödem kann die IPK eingesetzt werden. Sie reduziert das Ödem, vermin- } \\
\text { dert präoperativ angewendet die Infektionsrate und verbessert die Weichteilheilung und die Schmerzhaftig- } \\
\text { keit. }\end{array}$ \\
\hline Lymphödeme & $\begin{array}{l}\text { Die IPK kann eine adjuvante Therapieform zur komplexen physikalischen Entstauungstherapie (KPE) darstel- } \\
\text { len, insbesondere bei distal betonten Arm- oder Beinödemen - ohne Beteiligung des ipsilateralen Rumpfqua- } \\
\text { dranten - und bei eingeschränkter Mobilität des Patienten. } \\
\text { - Bei unter KPE nicht kompensierten Lymphödemen sollte die IPK additiv eingesetzt werden } \\
\text { - Die IPK kann bei Patienten mit Lymphödem als Heimtherapie eingesetzt werden, ärztliche Kontrollen sollten } \\
\text { garantiert sein. } \\
\text { - Zur Therapie des Lymphödems sollten Mehrstufengeräte am Bein mit intermittierenden Kompressionsdrücken } \\
\text { bis zu } 120 \text { mmHg abhängig von der Gewebebeschaffenheit und am Arm bis } 40 \text { mmHg angewendet werden. } \\
\text { Dabei sollten längere In- und Deflationszeiten (je um } 50 \text { s) und ein sequenzieller Druckaufbau gewählt wer- } \\
\text { den. }\end{array}$ \\
\hline Lipödeme & $\begin{array}{l}\text { Zur Therapie des Lipödems kann die IPK zur Ödemreduktion, Schmerzlinderung und Reduktion der Häma- } \\
\text { tomneigung - auch als Heimtherapie - eingesetzt werden. }\end{array}$ \\
\hline pAVK & $\begin{array}{l}\text { Bei einer pAVK mit stabiler Claudicatio intermittens oder kritischer Ischämie soll die Indikation zur IPK ge- } \\
\text { prüft werden. Voraussetzung für eine Therapie mit IPK ist jedoch, dass eine interventionelle oder operative } \\
\text { Rekonstruktion nicht in Frage kommt und ein angeleitetes Übungsprogramm nicht möglich ist. } \\
\text { Die IPK bei pAVK sollte durchgeführt werden mit Fuß und Wadenmanschette, Zielanpassdrücken von } 85 \text { - } \\
120 \text { mmHg, kurzer Inflationszeit, } 3 \text { Zyklen pro Minute und täglicher Anwendung. } \\
\text { Zur Reduktion des postoperativen Ödems kann die IPK nach einer operativ-rekonstruierenden Maßnahme bei } \\
\text { pAVK eingesetzt werden. }\end{array}$ \\
\hline Diabetische Fußläsion & - Keine konkrete Empfehlung bei fehlenden Daten \\
\hline $\begin{array}{l}\text { Hemiplegie mit sensorischer Störung } \\
\text { und Ödem }\end{array}$ & - Bei hemiplegen Patienten mit einer sensorischen Störung kann eine IPK zur Verbesserung eingesetzt werden. \\
\hline
\end{tabular}

\section{Patienteninformation}

Wie auch bei allen anderen Therapiemaßnahmen sind die Patienten auch für die IPK über Nutzen, Risiko und alternative Therapieoptionen eingehend zu informieren. Es empfiehlt sich, diese Information und Aufklärung sowie das Einverständnis oder die Ablehnung des Patienten zu dokumentieren.

Die IPK stellt entsprechend der aktuellen Datenlage in keiner Indikation eine unausweichliche, alleinige oder zwingend notwendige Maßnahme dar, doch hat sie in einigen Einsatzgebieten erhebliche positive Effekte. Entsprechend sollte auch aus forensischen Gründen die Verweigerung des Patienten, die Therapie durchführen zu wollen oder ein bewusster/ begründeter ärztlicher Verzicht auf diese additive Maßnahme, schriftlich dokumentiert werden. 


\section{Rechtliche Grundlagen, Normen und Verordnungen}

Die Geräte zur IPK sind als „Hilfsmittel zur Kompressionstherapie" in der Produktgruppe 17 des Hilfsmittelverzeichnisses gelistet (Stand 9/2015) und mit einer mehrstelligen Hilfsmittel-Positionsnummer versehen. Entsprechend können sie zu Lasten der Krankenversicherungen verordnet werden. Es werden Klinik-, Praxis und Heimgeräte unterschieden. Eine Normierung der Geräte gibt es bisher nicht und ist gegenwärtig auch nicht vorgesehen.

Die Geräte zur IPK können stationär oder ambulant in einer medizinischen Einrichtung eingesetzt werden. Darüber hinaus ist eine Anwendung mittels Heimgerät insbesondere bei langfristiger Indikation sinnvoll (z.B. pAVK, Lymphödem, CVI) und fördert das Selbstmanagement und die Eigenverantwortlichkeit des Patienten.

Vor einer Verordnung eines Heimgerätes sollte ein Wirksamkeitsnachweis vorliegen (Testphase in Klinik oder Praxis unter ärztlicher Aufsicht) und eine entsprechende Einweisung des Patienten bzw. seiner Angehörigen in den Umgang mit dem Gerät erfolgen. Auch in den Phasen der Heimanwendung sollten regelmäßige ärztliche Kontrollen erfolgen und der Patient eine klare ärztliche Anweisung über die Eckdaten der Therapie erhalten: Dauer pro Tag, Häufigkeit der Anwendung pro Woche bzw. pro Tag, Dauer der Anwendung insgesamt, Druckeinstellung sowie ggf. notwendige Schutzmaßnahmen zur Vermeidung von potenziellen Nebenwirkungen.

\section{Geräte}

Die IPK-Geräte bestehen üblicherweise aus 2 Komponenten - einem Steuergerät und den Manschetten. Dabei gibt es eine große Varianz an verschiedensten Gerätetypen im klinischen Einsatz, die sich unterschei- den hinsichtlich der Art der Kompressoren und Manschetten.

Das Steuergerät ist ein Medizinprodukt der Gruppe IIa und ist entscheidend für den Ablauf der Behandlung - Druckaufbau (intermittierend/sequenziell), Druckhaltephase, Ablassphase, Pausenzeiten und Zykluswiederholung.

Bei den Manschetten unterscheidet man nach Anzahl und Anordnung (einkammerig/mehrkammerig, überlappend/ nicht überlappend) der in den Manschetten befindlichen Kammern sowie der Art, wie diese befüllt werden (gleichzeitig oder nacheinander). Weiterhin unterscheiden sich die Manschetten abhängig von Applikationsort in Fuß- bzw. Fuß- und Unterschenkelmanschetten (ein Spezialschuh mit aufpumpbarer Sohle und ggf. auch Wadenmanschette), Extremitätenmanschetten (Beine oder Arme) sowie Hosen- und Jackenpumpen, die mittels doppelwandiger Behandlungsmanschetten Druck auf die Extremität bzw. den Körperstamm abgeben.

Oft kommt es zu Verwechslungen durch die Begriffe „Mehrstufen-“ und „Mehrkammer-Geräte“. Für die Behandlungsoptionen des Gerätes ist vor allem die Einstellung des Steuergerätes entscheidend, eine unterschiedliche Anzahl von Druckstufen zu erzielen und nicht die Kammeranzahl der angeschlossenen Manschetten. Entsprechend sollte dem Begriff „MehrstufenGeräte" Vorrang gewährt werden.

\section{Einsatz der IPK nach Indikationen}

Abhängig von den unterschiedlichen Indikationen, zu erreichenden Ziel-Gefäßsystemen und Zielgeweben (Lipödem, Thromboseprophylaxe, Lymphödem, pAVK) und der Gewebebeschaffenheit variieren die angewendeten Therapieprotokolle (à Geräte, Komponenten, Behandlungszyklus, Behandlungsfrequenz) bei der IPK erheblich.
Es gibt nur wenige Studien, die sich mit dem kontrollierten Vergleich verschiedener Gerätetypen und Behandlungsprotokolle beschäftigen. Es ergeben sich aus der Literatur und dem Expertenkonsens folgende Empfehlungen für die IPK nach Indikationen ( Tab. 4).

\section{Fazit für die Praxis}

Die vorhandene Datenlage zu den verschiedenen Indikationen ist sehr heterogen angesichts einer breiten Varianz an Geräten und Behandlungsprotokollen.

Zusammenfassend lässt sich auf der Basis der vorhandenen Literatur feststellen, dass die IPK bei richtiger Indikationsstellung und Anwendung - auch additiv - eine effektive und sichere Therapiemethode insbesondere in der Behandlung verschiedener Gefäß- und Ödemerkrankungen, der Wundheilung, der Thromboseprophylaxe sowie auch in der Therapie der pAVK darstellt, auch wenn nur RCTs mit meist geringer Evidenz vorliegen und die Empfehlungen teilweise auch empirisch sind.

\section{Interessenkonflikt}

S. Reich-Schupke: Honorare für Vortragsund Schulungstätigkeiten von Medi,

Sigvaris, Juzo und Ofa; C. Schwahn-Schreiber: Honorare für Vortrags- und Schulungstätigkeiten von Sigvaris, medi und Lymphologic

\section{Ethische Richtlinien}

Für das Manuskript wurden keine Studien an Menschen oder Tieren durchgeführt.

\section{Literatur}

Die Literaturliste entnehmen Sie bitte der Vollversion der Leitlinie aus den o.g. Quellen. 


\section{Zertifizierte Fortbildung}

Fragen zum Thema „Auszug aus der S1-Leitlinie IPK, AIK"

1. Was trifft auf die IPK zu?

A. Bei der IPK werden prophylaktisch oder therapeutisch pneumatische Wechseldrücke angewendet.

B. Die IPK wird ausschließlich prophylaktisch angewendet.

C. Die IPK wird nur stationär durchgeführt.

D. Die Geräte der IPK bieten keine Differenzierung nach Indikationen.

E. Die Wirkung der IPK ist rein mechanisch.

2. Was gehört nicht zu den Indikationen der IPK?

A. Extremitätenlymphödem

B. Ulcus cruris venosum mit fehlender Heilungstendenz unter konsequenter Kompressionstherapie

C. Thromboembolieprophylaxe

D. Periphere arterielle Verschlusskrankheit im Stadium I ohne Vortherapie

E. Ödeme anderer Genese, z.B. posttraumatisch

3. Was gehört nicht zu den absoluten Kontraindikationen der IPK?

A. Dekompensierte Herzinsuffizienz

B. Ausgedehnte Thrombose

C. Akute Entzündung (Erysipel/Phlegmone)

D. Neuropathie der Extremitäten

E. Kompartmentsyndrom

4. Welche Aussage zu den Risiken und Nebenwirkungen der IPK trifft nicht zu?

A. Nebenwirkungen der IPK sind bei korrekter Anwendung sehr selten

B. Die IPK gilt als sicher und effektiv

C. Am häufigsten sind Nebenwirkungen an der Haut, v.a. bei mehrtägiger kontinuierlicher Anwendung

D. Fragile Hautverhältnisse können Nebenwirkungen der IPK begünstigen

E. Genitallymphödeme sind auch in aktuellen Studien eine Hauptnebenwirkung der IPK

\section{Welche Aussage zur IPK trifft zu?}

A. Für die IPK ist eine schriftliche Einwilligung des Patienten erforderlich.

B. Es empfiehlt sich, die Aufklärung und Einwilligung zu dokumentieren.
C. IPK ist entsprechend der aktuellen Datenlage eine zwingend notwendige Maßnahme.

D. Die Geräte der IPK sind nicht im Hilfsmittelverzeichnis gelistet.

E. Die Geräte zur IPK bestehen aus 3 Komponenten - Generator, Manschetten und Druckmessgeräte.

6. Welche Aussage zur IPK trifft nicht zu?

A. Die IPK kann zu Lasten der GKV und PKV verordnet werden.

B. Geräte zur IPK können stationär, ambulant oder in Heimtherapie eingesetzt werden.

C. Die Heimanwendung empfiehlt sich v.a. bei langzeitigen Indikationen.

D. Während der Heimanwendung sind keine ärztlichen Kontrollen erforderlich.

E. Für die Heimanwendung sollte eine klare Anwendungsempfehlung gegeben werden.

\section{Was gilt nicht für den Einsatz der IPK} bei der $\mathrm{CVI}$ ?

A. Bei Patienten mit CVI kann die IPK eingesetzt werden, um chronisch venöse Symptome und die Lebensqualität zu verbessern.

B. Die IPK kann bei Patienten mit CVI als Heimtherapie eingesetzt werden.

C. Die IPK bei CVI ohne Ulcus cruris sollte mit Druckwerten von 30 - 40 mmHg, einer Inflation von 15s und einer Deflation von 10s mit mehrstufigen Manschetten.

D. Die IPK (Bein) sollte bei Patienten, deren UIcus cruris venosum unter Standardtherapie keine Heilungstendenz zeigt mit mehrstufigen Manschetten (sequentieller Druckaufbau, Zieldruck 40 - $50 \mathrm{mmHg}$, mindesten 1 Stunde pro Tag, mind. $3 \times$ pro Woche) zur Wundheilungsförderung eingesetzt werden.

E. Der Druckaufbau bei der CVI erfolgt von proximal nach distal.

\section{Was gilt nicht für die Anwendung der IPK beim Lymph- und Lipödem?}

A. IPK kann additiv bei der physikalischen Entstauungstherapie eingesetzt werden.

B. Die IPK sollte beim Lymphödem mit Mehrstufengeräten und sequentiellem Druckaufbau durchgeführt werden.

C. Die IPK ist als alleinige Maßnahme in der Therapie des Lipödems empfohlen.

D. Die IPK sollte ergänzend zu MLD und Kompressionsversorgung eingesetzt werden.

E. IPK ist beim Lipödem nicht kontraindiziert.
CME-Fortbildung online

Die Teilnahme an dieser durch die Ärztekammer Nordrhein zertifizierten CME-Fortbildung ist für 12 Monate ausschließlich online möglich. Zur Anmeldung gehen Sie bitte auf http://cme.thieme.de. Dort erfahren Sie auch den genauen Teilnahmeschluss. Es ist immer nur eine Antwort pro Frage zutreffend. Als Abonnent der Phlebologie nehmen Sie kostenlos am CME-Programm teil. Je nach CME-Fortbildung erhalten Sie bis zu drei Fortbildungspunkte. Weitere Informationen zur Anmeldung und Registrierung finden Sie unter http://cme. thieme.de. Sie erhalten bei inhaltlichen und technischen Fragen tutoriellen Support.

VNR 2760512018154652960

9. Was gilt nicht für die Anwendung der IPK bei der pAVK und dem Diabetes mellitus?

A. Bei einer pAVK mit stabiler Claudicatio intermittens oder kritischer Ischämie soll die Indikation zur IPK geprüft werden. Voraussetzung für eine Therapie mit IPK ist jedoch, dass eine interventionelle oder operative Rekonstruktion nicht in Frage kommt und ein angeleitetes Übungsprogramm nicht möglich ist.

B. Die IPK bei pAVK sollte durchgeführt werden mit Fuß und Wadenmanschette, Zielanpressdrücken von $85-120 \mathrm{mmHg}$, kurzer Inflationszeit, 3 Zyklen pro Minute und täglicher Anwendung.

C. Zur Reduktion des postoperativen Ödems kann die IPK nach einer operativ-rekonstruierenden Maßnahme bei pAVK eingesetzt werden.

D. Für die diabetische Fußläsion gibt es keine konkreten Empfehlungen bei fehlenden Daten.

E. Die IPK sollte als erste Maßnahme in der Therapie der pAVK im Stadium I eingesetzt werden.

\section{Was trifft für die IPK nicht zu?}

A. Die Datenlage zu den verschiedenen Indikationen ist sehr heterogen.

B. Es gibt eine erhebliche Varianz an Behandlungsprotokollen und Geräten zur IPK.

C. Viele Empfehlungen zur IPK basieren auf geringer Evidenz und/ oder Erfahrungswissen bzw. Expertenempfehlung.

D. Einsatzgebiete der IPK für die Therapie sind v.a. neurologische Erkrankungen.

E. Ein prophylaktischer Einsatz der IPK hat sich bei der Thromboembolie bewährt. 